Communication failure

\section{Why communication fails in the operating room}

\section{J Firth-Cozens}

\section{The reasons behind miscommunication need understanding in order to find the right solutions}

$\mathrm{T}$ here is evidence from a variety of sources that communications between members of healthcare teams emerge as a key factor in poor care and are especially apparent where medical errors occur. Lingard et $a l^{1}$ take this as their starting point for an observational study of communication failures in operating theatres published in this issue of QSHC. They found that $31 \%$ of all communications could be categorised as a failure in some waywhether the information was missing or the timing was poor, or where issues were not resolved or key people absent. Moreover, more than a third of these failures had negative effects on what was happening. If we multiply all that by the million patients treated daily in the UK alone, we are into an overwhelming amount of miscommunication. Lingard et al ${ }^{1}$ suggest training interventions to remedy this but, to make such training appropriate, the causes of poor communication need to be considered.

Some of these causes will be systemic. Team instability—-for example, different scrub nurses-can help to produce inferior outcomes in terms of care. ${ }^{2}$ This shows the importance of human resource management in ensuring good teamwork where members can know and understand each other well. Research in other fields has shown that the longer a team is together, the better its results, ${ }^{3}$ and at least part of this is likely to reflect improvements in communication. Organisational and team policies about communication are also important; ${ }^{2}$ a policy which disallows distraction in the operating room appears beneficial, probably because of the inevitable effects of distraction on communication.

Another systemic cause which is often ignored by research concerns resources. Having more than minimal staffingknown in high reliability organisations as "redundancy" - allows people the time to communicate properly, ${ }^{5}$ whether by ensuring that the timing is appropriate or that the person speaking can check that the receiver has understood what was said. Communication is not simply about transmitting but also receiving, including the knowledge that the transmission was understood in the way intended. Redundancy of communication may also be a concept to consider-team meetings outside the immediate task may seem like luxury in today's health services but it is from these "ordinary" interactions that rapport can be developed and communication improved.

Having sufficient resources in terms of staff and equipment will also have an indirect effect on communication by its effect on the stress levels of team members. No one communicates well when they are very stressed, and surveys consistently show that about $28 \%$ of clinical staff have stress symptoms above threshold levels. ${ }^{6}$ This human resource management issue demands both systems interventions in terms of staffing and support and interventions to increase individual resilience.

Other individual causes of poor communication centre round personality. For example, introverts (and there are many of them in hospital medicine ${ }^{7}$ ) may need training in making all the communications necessary and making them clearly. Extroverts, on the other hand, may need help in reducing their communications to those that are essential to the purpose. Personality may also be a factor in the extent to which leaders have the ability to encourage participative communication from team members, even when it may be to say that the leader has done something wrong. ${ }^{8}$ This ability to hear and even welcome negative messages is important in aviation safety and can be taught.'

One of the key reasons for miscommunication mentioned by Lingard et $a l^{1}$ arises from the power relationships that exist in health care as a result of different professional groups with traditionally different status, and of a culture where hierarchy still resembles the military model. Tannen ${ }^{10}$ has described how misunderstandings arise during conversations between people of different status and sex because of their very different ways of communicatingfor example, to demonstrate their status on the one hand, or to show their solidarity with colleagues on the other. There is a human benefit to this solidarity, but a similar benefit to retaining individual power. Professional language-sometimes difficult to understand by others-might be seen as a way of maintaining this power. The recognition of these differences is an important step towards changing them, but a difficult one since the conflicting human needs of involvement and independence may underlie them. The thinning of professional boundaries through shared training and other experiences should be a useful step towards improving communication.

The reasons behind miscommunication are therefore deeply complex. Changing it will require interventions at both the systems level and the individual level. However, any change is never likely to be permanent, so the checklist proposed by Lingard et al ${ }^{1}$ may well be an essential safety net for occasions when other more human interactions occur and communication fails.

Qual Saf Health Care 2004;13:327. doi: 10.1136/qshc.2004.010785

Correspondence to: Professor J Firth-Cozens, Special Advisor on Modernisation Postgraduate Medical \& Dental Education London Deanery, University of London, 33 Millman Street, London WCIN 3EJ, UK jfirth-cozens@londondeanery.ac.uk

\section{REFERENCES}

1 Lingard L, Espin S, Whyte S, et al. Communication failures in the operating room: an observational classification of recurrent types and effects. Qual Saf Health Care 2004; 13:330-4.

2 Carthey J, de Laval MR, Wright DJ, et al. Behavioural markers of surgical excellence. Saf Sci 2003;41:409-25.

3 Fouchee HC, Helmreich RL. Group interaction and flight crew performance. In: Weiner EL, Hagel DC, eds. Human factors in aviation. San Diego, CA: Academic Press, 1988:189-227.

4 Roberts K. Managing high reliability organizations. Calif Manage Rev 1990;32:101-13.

5 Firth-Cozens J. Teams, culture and managing risk In: Vincent C, eds. Clinical risk management.2nd ed. London: BMJ Books, 2001.

6 Firth-Cozens J. Interventions to improve physicians' well-being and patient care. Soc Sci Med 2001;52:215-22.

7 Clack GB, Allen J, Cooper D, et al. Personality differences between doctors and their patients: implications for the teaching of communications skills. Med Educ 2004;38:177-86.

8 Firth-Cozens J. Organisational trust: the keystone to patient safety. Qual Saf Health Care 2004;13:56-61

9 Helmreich RL, Willhelm JA. Outcomes of crew resource management training. Int J Aviat Psychol $1991 ; 1: 287-300$.

10 Tannen D. That's not what I meant!. London: Virago, 1992. 


\section{Prescribing how NHS trusts "do" quality: a recipe for committees but little action?}

\section{P M Whitty}

\section{The jury is still out as to whether the current clinical governance model is the best way to improve quality}

C inical governance, together with a number of national bodies to support and monitor it, was established in the NHS in 1997. ' Seven years on, it seems a good time to reflect on how effective this national quality assurance/improvement strategy has been, particularly in the light of subtle changes in the UK government's attitude to quality. The recent publication of a new standards framework, ${ }^{2}$ the replacement of the Commission for Health Improvement (CHI) with the Healthcare Commission from April 2004, and insistence that trusts will have greater local autonomy $y^{2}$ all suggest a change in the wind-although the direction of the change is not quite clear.

In the initial guidance on the duty of NHS trusts to implement clinical governance, ${ }^{34}$ the government set out the mandatory components of clinical governance (variously slightly modified since but broadly comprising clinical risk management, clinical audit, patient/service user involvement, education and training, clinical effectiveness and research and development, staff focus, and use of information) and some of the structures that must be in place (notably, a senior clinician on the trust board and a board level clinical governance committee). Not surprisingly then, almost all trusts now have these mandatory structures (see www.chi.nhs.uk for archived CHI clinical governance review reports) and many other committees and systems besides, with structures often mirroring the "seven pillars" of clinical governance prescribed in the national guidance. But what evidence is there that such structures improve the processes and outcomes of care? And could a "one size fits all" approach to structures even mitigate against such improvements? This has been a massive-and, no doubt, costly-initiative and other countries would do well to examine the UK experience in depth before embarking on any similar approaches.

Robust evaluations of the clinical governance initiative are very much needed. As Freeman and Walshe report in their paper in this issue of $Q S H C$, ${ }^{5}$ evaluations of clinical governance implementation to date have tended to focus on implementation of structures. Their survey begins to address the issues of process and outcomes, and to distinguish between success in quality assurance and quality improvement dimensions. While their survey is necessarily limited to perceptions and does not include those of clinicians, it nevertheless begins to ask the questions that really matter. What they find is not overly encouraging, supporting earlier evidence that structures are now well embedded and requirements of quality assurance fulfilled, but finding little priority given to or progress in quality improvement.

Of course, evaluation of such a wide ranging policy initiative as clinical governance is challenging. It may be difficult to determine what the appropriate process and outcome improvements should be; organisation-wide programmes such as these are very difficult to evaluate in traditional study designs; ${ }^{6}$ and it may be impossible to identify adverse consequences that have been averted, for example, through the application of good risk management systems. It could also be said that some components-for example, those related to reducing the consequences of litigation-are good "business" practice for any organisation or that others like patient/service user involvement are a duty of public sector organisations. However, the core problem remains: setting overall priorities and policy is an appropriate task for government, while prescribing the detail of how these issues should be tackled at a local level is frequently counterproductive. ${ }^{78}$

Few would disagree that the assurance and continuous improvement of the quality of health care for people in the UK is an essential duty of all healthcare organisations. As to whether the current clinical governance model is the way to do it, the jury is still out. No doubt there are selected components within the model that may be more promising than others-for example, research on the safety agenda is being heavily invested in via the NHS R\&D Patient Safety Research Programme, allowing the National Patient Safety Agency (www.npsa.nhs.uk) to investigate and apply an evidence base for this part of the initiative. By contrast, the UK still seems determined to promote an approach to clinical audit that has been shown to be limited at best. ${ }^{910}$ If the UK is really coming (back) to devolving more control to local organisations, isn't it time to monitor their success in improving quality through their process and outcomes rather than their structures? The new Healthcare Commission avows that this is their mission (www. healthcarecommission.org.uk): whether they can achieve it remains to be seen.

Qual Saf Health Care 2004; 13:328. doi: 10.1136/qshc.2004.011767

Correspondence to: Dr P M Whitty, Centre for Health Services Research, University of Newcastle, Newcastle upon Tyne NE2 4HH, UK; p.m.whitty@ncl.ac.uk

\section{REFERENCES}

1 Department of Health. The new NHS: modern and dependable. London: HMSO, 1997.

2 Department of Health. National standards, local action. Health and social care standards and planning framework 2005/06-2007/08, 21 July 2004.http://www.publications.doh.gov.uk/ planningframework0506/index.htm.

3 Department of Health. A first class service: quality in the new NHS. London: HMSO, 1998.

4 Department of Health. Clinical governance in the new NHS, HSC 1999/065. London: HMSO, 1999.

5 Freeman T, Walshe K. Achieving progress through clinical governance? A national study of health care managers' perceptions in the NHS in England. Qual Saf Health Care 2004:13:335-43.

6 Ovretveit J, Gustafson D. Using research to inform quality programmes. $B M$ 2003;326:759-61.

7 Chapman J. System failure: why governments must learn to think differently. London: Demos, 2002.

8 Sneddon J. Freedom from Command \& Control: a better way to make the work work. London: Vanguard Education, 2003.

9 Walshe K, ed. Evaluating clinical audit: past lessons, future directions. London: Royal Society of Medicine Press, 1995.

10 Jamtvedt G, Young JM, Kristofferson DT, et al. Audit and feedback: effects of professional practice and health care outcomes (Cochrane Review). The Cochrane Library 2004;3 
Right care

\section{Making sure the right patient gets the right care}

\section{A Ranger, S Bothwell}

\section{Preventing errors in patient treatment-right patients right care}

O ne of the main areas where the NHS needs to improve patient safety is the reduction and, where possible, elimination of errors in the matching of patients with their care. There are no accurate figures on the frequency or cost of such mismatching errors but we know from the evidence that is available that they account for a significant proportion of errors made in healthcare. Patient safety incidents can occur, for example, when a patient is given the wrong treatment as a result of a failure to match samples, specimens, or $x$ rays; when a patient is given the wrong treatment as a result of a failure in communication or checking; or when one patient is given treatment intended for another as a result of a failure to identify him or her correctly.

In 2000, an expert group chaired by Sir Liam Donaldson, the Chief Medical Officer, noted that adverse events occur in around $10 \%$ of NHS admissions or at a rate of about 850000 patient safety incidents a year. ${ }^{1}$ Around half of these incidents are preventable. The UK is not alone in this because research has shown similar rates for patient safety incidents in the US, Australia, Canada, and Denmark. As a result of the expert group's recommendations, the National Patient Safety Agency (NPSA) was established as a special health authority in England and Wales in July 2001. The NPSA's role is to improve the safety of NHS patients by promoting a culture of learning and reporting from patient safety incidents, and to manage the national reporting system to support this function.

Errors in making sure the right patient gets the right care can have a range of consequences. Some errors result in lasting but relatively minor consequences for the patient. Some, however, result in serious, lasting harm, such as chronic pain, undiagnosed cancers, blindness, and even death. For example, between 1996 and 2002 five patients are known to have died directly as a result of being given blood intended for others and six are thought probably to have done so. Sixteen others given the wrong blood died for reasons unconnected with the transfusion. ${ }^{2}$
The NPSA is convinced on the basis of the research it has commissioned (Mismatching between planned and actual treatments in medicine-manual checking approaches to prevention, Human Reliability Associates, 2004; and Ensuring patients are correctly matched with samples or specimens taken from them and treatment planned for them, Cambridge Consultants, January 2004. To be published by NPSA, http://81.144.177.110) that there is considerable scope in the NHS for improving patient safety by addressing mismatching of care. There is more than one way of approaching this. It could be done through the development of more fail-safe methods of manual identification and checking that does not entail the use of electronic technologies. It could also be approached through the application of modern technologies. The three most likely technologies are: barcoding, which is the most familiar form of ID coding technology using adjacent bars and spaces to present information; radio frequency identification, which uses radio frequency transfer of data between a reader and a tag; and biometrics, which uses automated methods of identifying or authenticating a living person based on physiological or behavioural characteristics.

Whatever system is used to ensure better matching of patients to care, it is highly likely that the patient will need to wear some form of identification bracelet (wristband) or label. The wristband should have printed personal details such as full name, date of birth, hospital number, and ward. However, in addition, information about the patient and their care may be contained in or accessed through a machine readable barcode on the wristband or, in due course, a radio tag in the wristband. A biometric patient identifier, such as an iris scan, could also be coded into a unique number and worn as a barcoded wristband. Thus, the advent of new technologies for matching patients to their care will not make the wristband redundant, but in fact enhance its role and make it increasingly important that wristbands are worn by hospitalised patients.

In some European countries, such as Switzerland, ${ }^{3}$ the wearing of wristbands is not systematic and this could make it more difficult to introduce manual and technological innovations to help make sure the right patient gets the right care. Although wearing a wristband as an inpatient is an accepted part of the system in the UK, this does not mean that patients always wear them, or that they are replaced when removed for surgery or procedures such as inserting an intravenous cannula. For example, an audit ${ }^{4}$ carried out at Guy's and St Thomas' NHS Trust in July 2000 showed that 34\% of patients were not wearing wristbands.

Psychiatric inpatients do not currently wear wristbands in the UK and some people with learning difficulties, mental illness, or personality disorders may be distressed by and resist any attempt to introduce a wristband. Neonates are often too small to retain a wristband and children can be keen to remove them. For patients cared for in the community, wearing a wristband could adversely affect their privacy and dignity.

Wristbands are not always used consistently by staff as part of a systematic process for checking they have the right patient for the treatment or care they are about to give. A recent study showed that $18 \%$ of patients receiving blood transfusions did not have their identity checked by staff when the pre-transfusion blood sample was taken (Safe and effective transfusion in Scottish hospitals the role of the transfusion nurse specialist. Gray A, Buchanan S, McClelland DBL, (2003) Unpublished report).

The NPSA is now looking into the use of wristbands as part of its wider initiative on making sure the right patient gets the right care. We will report on this with recommended action for the NHS next year.

Qual Saf Health Care 2004; 13:329. doi: 10.1136/qshc.2004.012005

\section{Authors' affiliations}

C A Ranger, S Bothwell, National Patient Safety Agency, 4-8 Maple Street, London, WIT 5HD

Correspondence to: C A Ranger, Head of Safer Practice, National Patient Satety Agency, 4-8 Maple Street, London, WIT 5HD; chris. ranger@npsa.nhs.uk

\section{REFERENCES}

1 Department of Health. An organisation with a memory, Report of an expert group on learning from adverse events in the NHS. London: Stationery Office, 2000.

2 Serious Hazards of Transfusion Organisation. Annual report 2001-2002, Serious hazards of transfusion. Manchester: SHOT, 2003.

3 Cleopas A, Kolly V, Bovier PA, et al. Acceptability of identification bracelets for all hospital inpatients. Qual Saf Health Care 2004; 13:344-8.

4 Guthrie D. Positive patient identification. Frontiers in Laboratory Medicine, London: The Royal College of Physicians, 2003 